Rev. Sci. Tech. Off. Int. Epiz., 2019, 38 (3), ... - ...

\title{
Contagious agalactia: costs and control revisited
}

This paper (No. 28112019-00158-EN) has been peer-reviewed, accepted, edited, and corrected by authors. It has not yet been formatted for printing. It will be published in December 2019 in issue 38 (3) of the Scientific and Technical Review.

G.R. Loria (1), R. Puleio (1), G. Filioussis (2), R.S. Rosales (3) \& R.A.J. Nicholas ${ }^{(4)}{ }^{*}$

(1) Area Diagnostica Specialistica, OIE Reference Laboratory for Contagious Agalactia, Istituto Zooprofilattico Sperimentale della Sicilia, Via G. Marinuzzi 3, 90129 Palermo, Italy

(2) Laboratory of Microbiology and Infectious Diseases, Faculty of Veterinary Medicine, Aristotle University of Thessaloniki, 54124, Thessaloniki, Greece

(3) Unidad de Epidemiología y Medicina Preventiva, Instituto Universitario de Sanidad Animal y Seguridad Alimentaria, Universidad de Las Palmas de Gran Canaria, C/Trasmontana s/n, Arucas, 35413 Gran Canaria, Spain

(4) The Oaks, Nutshell Lane, Farnham, Surrey GU9 0HG, United Kingdom

*Corresponding author: robin.a.j.nicholas@gmail.com

\section{Summary}

The economic costs of contagious agalactia (CA) to the small ruminant dairy industry are not well known but include losses due to mortality, lowered milk production, spoiled products, abortions and animal welfare problems, as well as diagnosis and treatment. This paper reports financial estimates made in Southern Europe, including a study on small- and large-scale farming systems in Italy, indicating that the financial losses are high and underestimated. Furthermore, the current control strategies, including chemotherapy and vaccination, in selected countries in Europe are described. In some countries, disease control is 
hampered by excessively strict veterinary legislation which discourages farmers and private veterinarians from notifying outbreaks because it leads to prohibition of milk sales and can result in delays in lifting restrictions. Furthermore, new European Union legislation may downgrade the importance of CA, which will have implications for international research efforts. Finally, a series of recommendations are provided that cover the proper notification and handling of CA outbreaks, including movement control, current diagnostics, treatment, vaccination and disinfection.

\section{Keywords}

Contagious agalactia - Economics - Husbandry - Legislation Mycoplasma agalactiae.

\section{Introduction}

Contagious agalactia (CA), a disease of dairy sheep and goats mainly caused by Mycoplasma agalactiae, has been present in Europe for over 200 years (1). The disease is found wherever sheep and goats are kept, but is concentrated in countries surrounding the Mediterranean Sea and western Asia (2). It is difficult to estimate the full scale of the problem because few countries have carried out prevalence studies. Data compiled by the World Organisation for Animal Health (OIE) provide some indication, although not all countries report the number of outbreaks, probably because they do not have mycoplasma diagnostic laboratories, while others, including France, Turkey and Portugal, just declare that the disease is present (3). The countries that reported outbreak numbers over a four-year period ending in 2018 include Iran with 968 outbreaks, Mongolia with 380, Italy with 254, Spain with 85, Greece with 17 and Israel with 2. These figures probably represent an underestimate because of the difficulty in detecting infected flocks and herds, many of which may not show overt clinical signs, and the reluctance of farmers/veterinarians to report outbreaks (1).

Although it can present as a mild disease in small ruminants, CA has proved difficult to eradicate because it may persist on farms, contaminating succeeding flocks (2). The main reservoir of the 
causative agent is the infected animal, in which mycoplasmas can persist for over one year after clinical recovery. Lambs and kids become infected directly at suckling while adults are contaminated via milkers' hands or milking machines. There is also evidence for transmission by aerosol over short distances and from infected bedding (2).

Unusually for an OIE-listed disease, four agents, all mycoplasmas, are listed as causing a clinically indistinguishable syndrome although M. agalactiae accounts for more than $80 \%$ of the disease outbreaks (2). The other three pathogens, $M$. mycoides subsp. capri, M. capricolum subsp. capricolum and $M$. putrefaciens, are more sporadic and are mainly found in goats.

The disease is first noticed when milk production falls, usually a few days after the introduction of healthy carriers or mixing with affected herds at pasture, markets or water sources (1). Milk becomes abnormal in appearance, mastitis develops and then production ceases in one or both halves of the udder, occasionally permanently. Keratoconjunctivitis and arthritis are chronic sequelae which are particularly severe in young animals and prevent them from keeping up with the rest of the group during transhumance and other animal movements. Laboratory tests are necessary to confirm a diagnosis because clinical signs are not specific. Mycoplasma isolation and/or polymerase chain reaction (PCR) tests are recommended for the detection of the pathogen and serological tests, in particular the enzyme-linked immunosorbent assay (ELISA), can facilitate rapid diagnosis from serum or milk (2).

This paper reviews the economic impact of the disease and current control strategies in selected countries of Europe, and provides a series of recommendations for the control of CA.

\section{Economic impact}

The financial losses due to CA as a result of mortality, diagnosis and treatment, lowered milk production, spoiled products, abortions and animal welfare problems are not well known. Previously the impact of the disease was thought to be most severe on small-scale farmers using 
traditional husbandry to make milk products (1). However, the disease may also have a major impact on larger commercial farms, though information is lacking.

\section{Greece}

In 1996, Legakis (4) made the first attempt to estimate the economic losses due to acute CA in Greece, using official data for the period 1994-1997. The losses, made up of milk reduction, abortions, neonatal deaths and dead animals, were estimated at $€ 24.5$ million per year. However, the author stated that these losses were most likely underestimated owing to the compulsory reporting of the disease in Greece, which leads to severe restrictions and results in farmers often not reporting affected herds. Furthermore, the impact of subacute or chronic disease, which causes physical weakness and clinical complications, is very hard to quantify.

\section{Spain}

In an effort to estimate the losses due to CA in Spain, Valergakis et al. (5) carried out a cost-benefit simulation of vaccination based on clinical experience and published data. They estimated average losses of approximately $€ 70$, $€ 83$, $€ 95$, $€ 108$ and $€ 120$ per ewe producing 150, 200, 250, 300 and $350 \mathrm{~kg}$ of milk per year, respectively. Milk losses accounted for $66.4 \%$ of the total costs associated with CA, followed by the cost linked to mortality/culling (31.3\%) and disease treatment costs $(2.3 \%)$. The costs of vaccination, based on two doses per ewe per year, were about the same as the losses due to CA. However, the efficacy of the commercially available vaccines is highly variable so improved vaccines would reduce costs further (6).

\section{Italy}

The losses due to CA were studied in two different farming systems in Sicily: a large commercial farm of mixed small ruminants and a smaller family farm. The first farm was located in the Enna district and comprised over 1,000 sheep and goats. Milk production dropped suddenly from 650 litres per day to approximately 100 litres per day in 
October 2015 and 10-15 animals developed clinical signs of CA on a daily basis. The course of the infection stopped in January 2016, following two vaccinations with an inactivated CA vaccine and because the animals entered the dry period. The disease had entered the farm through the introduction of a group of 120 apparently healthy Etna goats in March 2015. These goats were newly lactating animals without any unusual signs until July 2015 when the new parturition season began; at that time, 18 goats delivered weak kids and there was an unexpected drop in milk production. The goats had not been screened for CA before introduction to the farm. After this, clinical signs began to develop in the herd and by September CA had spread to $30 \%$ of the 1,000 susceptible ewes.

Following notification of the disease, the costs incurred included: the slaughter of 200 sick sheep and all 240 goats; vaccination of the remaining animals; and purchasing of replacement animals and milk from other farmers to maintain the dairy business. Although the disease appears to have been controlled on this farm the cost of the disease to the farmer was estimated at over $€ 100,000$ (Table I).

\section{Table I}

Economic losses caused by contagious agalactia on a large commercial farm of over 1,000 small ruminants

\begin{tabular}{lcc}
\hline Item & Number & Cost (euros) \\
\hline Sheep lost & 200 & 26,000 \\
Goats lost & 240 & 50,000 \\
Lambs and kids affected & 400 & 20,000 \\
Cost of extra milk & 400 litres & 25,000 \\
Vaccine & 30 vials & 2,550 \\
Total & & approx. 123,550 \\
\hline
\end{tabular}

The second, smaller, farm, located in the Palermo district, consisted of 200 sheep and goats. The outbreak was confirmed on May 2016 when milk production dropped by $27 \%$ from 150 to 110 litres per day. 
Twenty ewes were reported to be showing clear signs of CA. The clinical course of the infection stopped in June 2016 when all animals, after a single dose of inactivated CA vaccine, became dry. Surprisingly there were no other losses, probably because the disease developed on the farm around the time of the dry period (from May to June 2016); consequently, the pathogen did not have the opportunity to infect all the ewes during the period of lactation. To reduce the spread of the disease, the 20 sick sheep and 15 sick goats were culled. The purchase of replacement lactating animals and the use of vaccination to control the disease caused losses of several thousand euros to the farmer (Table II).

\section{Table II}

\section{Economic losses caused by contagious agalactia on a small family farm of 200 small ruminants}

\begin{tabular}{lcc}
\hline Item & Number & Cost (euros) \\
\hline Sheep lost & 20 & 2,600 \\
Goats lost & 15 & 1,950 \\
Lambs and kids affected & $1-2$ & scant \\
Losses of milk & 40 litres & 1,680 \\
Vaccine & 9 vials & 370 \\
Total & & approx. 6,600 \\
\hline
\end{tabular}

As demonstrated on these Sicilian small ruminant farms, the economic costs of CA outbreaks that are incurred by individual farmers are substantial, comprising the loss of animals and dairy production as well as the additional expenditure on disease control and restocking. Both farms will continue to vaccinate for three to four years in order to reduce the pathogen burden and clinical disease in the flocks and, ultimately, to protect the new stock of lactating ewes that are highly susceptible to the infection. Extrapolated over the whole of Sicily, with almost 8,700 farms, among which the authors estimate that $6 \%$ are affected (which is probably an underestimate because many animals are chronically infected and therefore difficult to identify), this represents a major burden on the local economy. 


\section{Good practice}

\section{Chemotherapy}

The main impediment to successful control of CA is the lack of awareness and international agreement on the way forward. France favours culling of affected flocks whereas Italy, Spain and Greece use a mixture of antimicrobials and vaccination (7). Despite a reported small increase in the minimum inhibitory concentrations of some antibiotics, in particular tylosin, over the last decade (8), in vitro data show that most antibiotics still appear to be effective against $M$. agalactiae $(9,10)$. This is in sharp contrast to the closely related pathogen of cattle, Mycoplasma bovis, against which, because of their widespread use in this industrial sector, virtually all classes of antimicrobial agents are poorly effective (11). However, the actual experience of antibiotic usage on small ruminant farms is much less satisfactory, and their use, while bringing about a quick clinical improvement, leaves long-term shedders that contaminate the environment and pose risks to susceptible animals, as well as increasing the risk of antimicrobial resistance.

\section{Vaccination}

Vaccination also leaves a lot to be desired. The majority of the vaccines used today are formalin-inactivated whole cell vaccines, containing $M$. agalactiae and, sometimes, one or more of the other causative mycoplasmas, with limited or no published efficacy. One inactivated vaccine could not protect sheep from natural challenge despite annual vaccinations over the previous three years (12). Furthermore, in a small trial, no potency was evident after vaccination using a commercial vaccine followed by contact challenge (6). Interestingly, a live attenuated vaccine, successfully used in Turkey for many decades, was safe and protective (6). While larger trials on commercial products are necessary, consideration should be given to using live vaccines (which are currently not allowed in Europe) in endemically affected areas. Another advantage is that this live vaccine does not provoke an antibody response as detected by ELISA, so vaccine antibody could be distinguished from natural infection (6). The development of DNA 
vaccines and recombinant vaccines has been reported but they have not yet been tried in the field (13). Sadly, based on developments in other areas of animal disease, there is little evidence that these innovative products will greatly improve the health of small ruminants affected with CA (7).

\section{National control plans}

In Europe, there are few targeted official programmes for the control of CA in operation today. However, the disease is not notifiable in Portugal, which is affected but has no plans for control. The current plans of a selection of European countries are outlined below.

Italy

Despite the endemicity confirmed by the massive sales of vaccines and antibiotics directed at mycoplasma infections, mostly CA, the number of outbreaks reported officially in southern Italy is remarkably low. Only a handful of outbreaks have been reported between 2012 and the present day. Consequently, the present numbers of reported outbreaks represent a large underestimate. The disease itself is not a zoonosis and is relatively easy to control because it is only spread by direct contact or via contamination by the hands of milkers or associated dairy equipment. In addition, it is not believed that the environment plays a large role in the epidemiology of the disease, particularly as the pathogen is sensitive to commonly used disinfectants. Similarly, vectors, particularly blood-sucking parasites, are not thought to be involved in transmission of CA, though this route of transmission has not yet been fully investigated.

Simple improvements in biosafety on farms are of course necessary but this requires, initially, an education programme for farmers. As discussed above, antibiotics are mostly effective and annual vaccination with approved vaccines can hold the disease in check. Unfortunately, the inability to control CA in some parts of Italy is largely the result of unnecessarily strict local veterinary legislation which discourages farmers and veterinarians from reporting outbreaks. The prohibition of selling milk and delays in lifting restrictions can have a serious impact 
on the income of small farmers (7). These restrictions, like the declaration of infected areas, which can cover a $3 \mathrm{~km}$ area in Italy and often involve adjoining farms that will suffer the same restrictions, are more appropriate to more serious diseases such as sheep pox and foot and mouth disease. The result of these restrictions is to discourage the notification of outbreaks, as can be seen in the under-reporting in official statistics.

More recently, the introduction of European Union (EU) regulation 2016/429, which provides a new regulatory framework covering control and surveillance of infectious diseases, animal welfare, certifications and animal movements amongst Member States, complements local legislation but also reduces the amount of intervention possible (14). Worryingly, there are proposals that some traditionally notifiable diseases, including CA and enterotoxaemia of sheep and goats, will be removed from the new EU disease listing process, with control being devolved, presumably, to the national or even local level. This apparent downgrading of the importance of CA may negatively affect international collaboration on control across borders and make this disease a less likely area for research funding.

\section{United Kingdom}

In the United Kingdom (UK), like many other disease-free countries, CA is a notifiable disease and is covered by two pieces of legislation: the Specific Diseases (Notification and Slaughter) Order 1992 and the Specific Diseases (Notification) Order 1996 which describe procedures to be taken, including movement restriction, slaughter and disinfection, in the event of an outbreak. These laws were put into action following the notification of the first detection of M. agalactiae in the UK in a small group of goats at a farm in mid Wales in 2014, which resulted in culling of the infected and contact animals (15).

\section{Spain}

A recent attempt was made to set guidelines for the future control of CA in Spain by the Ministry of Agriculture, Fisheries and Food, who have developed the first national voluntary programme for its control 
and eradication in sheep and goats (16). The programme, which includes detailed biosecurity, quarantine and parasite control measures, classifies existing farms into four types. The first includes CA-free farms which have obtained negative results in the last two annual screening rounds, where vaccination is not performed and where all animals introduced after the first testing have come from herds officially free from or negative for CA. Here, all animals older than four years will be tested annually by serum ELISA and PCR on ear swabs or nasal swabs. The second type comprises farms that are officially free of disease but where vaccination is still carried out. Ear or nasal swabs from male animals on these farms will be screened by PCR once a year. Additionally, on milk production farms, five milk tank samples will be tested by PCR twice, at two-month intervals, with the first one being taken after 15 days from the beginning of lactation. On meat farms, in addition to the samples from males, 14 samples will be taken from lambs or kids for testing by ELISA and PCR. The third group of farms comprises those that have obtained negative results on testing during their first year of participation in the programme and will subsequently become subject to regular screening. Finally, the fourth group contains those with no participation in the official surveillance programme. Although in the short term there are no immediate benefits to farms joining the voluntary programme, owing to the additional costs of testing, in the longer term, when it is likely to become compulsory, farms will be certified as CA-free with the benefits of additional income from this new status.

Control of CA requires that the biosafety measures taken on the farm are flexible and appropriate to the type of production and the species concerned; they must also take into account local circumstances and, importantly, must be shared with the competent authorities. Moreover, in the light of a policy of prevention of communicable diseases, the EU encourages the development of local guidelines for good practice and/or special rules for diseases, such as CA, which are considered 'less dangerous' but still have a large local economic impact. It is therefore the duty of responsible bodies to provide veterinarians and field operators with guidelines or good practices that, on the one hand, 
respect the existing regulations and, on the other hand, take into account the level of risk and the epidemiological factors of the area involved.

\section{France}

Numerous outbreaks of CA in France in the 1990s justified the introduction of a preventative health programme, begun in 1998, which relied on herd certification, mandatory reporting and quarantine. This was effective in reducing outbreaks to almost zero between 2003 and 2006, but in 2007 a resurgence of the disease was observed with 190 new outbreaks notified in 2007-2009 (17). New outbreaks were also seen in Corsica in 2010 (7). Consequently, more effective measures, including vaccination, were introduced to improve control (18). To date, CA caused by M. agalactiae has largely been eradicated from all but the south-western region of France, where it stubbornly persists.

\section{Greece}

In Greece, legislation was introduced in 2006 to reduce the incidence of CA (19). Farmers voluntarily submitted requests to be included in an official scheme to show that their holdings were free from CA, which would be enabled by free laboratory testing. Owners whose flocks and herds are affected by CA are compensated for the value of slaughtered animals, the loss of milk production and associated costs of the precautionary farm quarantine period. In 2010, because the incidence of CA in goats was found to be higher than that in sheep, the control plan was modified to eradicate CA in livestock units with goats only and in mixed livestock units with more goats than sheep. Despite these efforts to control CA there is anecdotal evidence that cases have increased over the last decade (unpublished results). There are also concerns that commercial vaccines are not as effective in goats as they are in sheep, but this needs further investigation.

\section{Recommendations}

It is recommended that whenever an outbreak is confirmed the competent authority and farmer must be informed and this properly recorded. Susceptible animals should be identified, and animals should 
be prevented from leaving and entering the infected farm/holding or, if the area is difficult to define, all land within $1 \mathrm{~km}$ surrounding the affected farm. The culling of infected animals should be encouraged to occur at a slaughterhouse that follows normal biosecurity arrangements or has been quarantined. Milk from affected animals should be prohibited from purchase or heat treated on the premises if destined for food animals. Milk from unaffected animals should only be used if permission is granted by the competent authority. All forms of transport used for the animals, shelters, resting areas, milking areas, milk storage areas, tools and utensils, with particular reference to those destined to come into contact with milk, should be disinfected following standard procedures and using authorised products.

Infected and suspect animals on the affected farm should be isolated immediately in a quarantine area to prevent contact with other animals. Treatment with the most appropriate antibiotic should be carried out under the instructions of a veterinarian. In addition, vaccination of the entire flock, including affected animals, with a licensed inactivated vaccine should be mandatory, with two doses given at a 15-day interval followed by another two doses six months apart, repeated for at least two to three years. As the pathogen and antibody may persist in subclinically affected or healthy carriers for over one year, even in vaccinated animals, the control measures should be maintained until the mycoplasma is completely cleared from bulk milk or, preferably, individual milk samples. This can be confirmed by negative results in two repeated PCR tests under the supervision of the veterinarian. Stricter controls should be put in place for animals exported to CA-free countries.

\section{Conclusions}

Estimating the economic impact of CA is complex, probably because it is connected with traditional husbandry typical of Mediterranean areas; invariably, however, CA has a strong impact on the local economy because of its high morbidity rate, which is why it is often known as 'the shepherd's nightmare' (7). Moreover, owing to the indirect effect on the quality of milk produced, it is likely that the impact of CA is 
underestimated, especially in dairy livestock. In this paper the economic costs of CA were estimated on two different farm types: a small family farm and a large commercial holding. Extrapolated across southern Italy, where most of these farms are located, these figures represent significant losses to this poorer sector where there is little or no farmer compensation. Some recommendations have been made to improve the control of this disease, although its impact could be reduced by the EU's proposals to downgrade the disease control to national or even local importance. However, such a development could affect cross-border control and make this a less attractive area for research funding. Many measures are still necessary to improve control of this disease. They include: the development of a safe vaccine which provides greater and longer-lasting protection; reliable screening tests, such as pen-side tests which can assess the infection status of a herd quickly; and, finally, a greater awareness among national and international authorities of the true social and economic impact of this disease.

\section{References}

1. Nicholas R., Ayling R. \& McAuliffe L. (2008). - Contagious agalactia. In Mycoplasma diseases of ruminants (R. Nicholas, R. Ayling \& L. McAuliffe, eds). Chapter 8, CABI, Oxfordshire, United Kingdom, 98-113. https://doi.org/10.1079/9780851990125.0098.

2. World Organisation for Animal Health (OIE) (2018). Contagious agalactia. In Manual of Diagnostic Tests and Vaccines for Terrestrial Animals. Chapter 3.7.3. OIE, Paris, France, 1430-1440. Available at: www.oie.int/fileadmin/Home/eng/Health_standards/ tahm/3.07.03_CONT_AGALACT.pdf (accessed on 5 May 2019).

3. World Organisation for Animal Health (OIE) (2019). World Animal Health in 2012. In World Animal Health Information Database (WAHIS Interface). OIE, Paris, France. Available at: www.oie.int/wahis_2/public/wahid.php/Diseaseinformation/statusdeta il (accessed on 2 March 2019).

4. Legakis K. (1996). - An economic appraisal of the acute form of contagious agalactia in the transhumant sheep and goats in 
Greece. In Mycoplasmas of ruminants: pathogenicity, diagnostics, epidemiology and molecular genetics (J. Frey \& K. Sarris, eds). EUR 16934, COST, European Commission, Brussels, Belgium. European Communities Official Publications Office, Luxembourg, 100-103.

5. Valergakis G.E., Termatzidou S.A., Gelasakis A.I. \& Arsenos G. (2015). - Cost-benefit simulation analysis of vaccination against contagious agalactia of dairy sheep in Greece. In Proc. British Society of Animal Science (S. Athanasiadou, A.S. Chaudhry [...] \& A.R.G. Wylie, eds), 14-15 April 2015, Chester. Cambridge University Press, Cambridge, United Kingdom, 6 (S2), 89. Available at: www.cambridge.org/core/journals/advances-in-animalbiosciences/issue/A9D1925C7093DCA119097BED1000D985 (accessed on 10 October 2019).

6. Agnone A., La Manna M., Sireci G., Puleio R., Usticano A., Ozdemir U., Nicholas R.A.J., Chiaracane V., Dieli F., Di Marco V. \& Loria G.R. (2013). - A comparison of the efficacy of commercial and experimental vaccines for contagious agalactia in sheep. Small Rum. Res., 112 (1-3), 230-234. https://doi.org/10.1016/ j.smallrumres.2012.12.022.

7. Loria G.R. \& Nicholas R.A.J. (2013). - Contagious agalactia: the shepherd's nightmare. Vet. J., $198(1)$, 5-6. https://doi.org/10.1016/j.tvjl.2013.06.017.

8. Prats-van der Ham M., Tatay-Dualde J., de la Fe C., Paterna A., Sánchez A., Corrales J.C., Contreras A. \& Gómez-Martín Á. (2017). - Molecular resistance mechanisms of Mycoplasma agalactiae to macrolides and lincomycin. Vet. Microbiol., 211, 135-140. https://doi.org/10.1016/j.vetmic.2017.10.012.

9. De Garnica M.L., Rosales R.S., Gonzalo C., Santos J.A. \& Nicholas R.A.J. (2013). - Isolation, molecular characterization and antimicrobial susceptibilities of isolates of Mycoplasma agalactiae from bulk tank milk in an endemic area of Spain. J. Appl. Microbiol., 114 (6), 1575-1581. https://doi.org/10.1111/jam.12176. 
10. Marne J., Poumarat F. \& Tardy F. (2019). - Antibiotic resistance in small ruminant mycoplasmas: first steps towards the definition of epidemiological cut-offs. In Abstracts of the European Mycoplasma Conference, 18-19 March 2019, London. Public Health England, London, United Kingdom, Abstract 39, 96.

11. Nicholas R.A.J. (2011). - Bovine mycoplasmosis: silent and deadly. Vet. Rec., 168 (17), 459-462. https://doi.org/10.1136/vr.d2468.

12. Leon Vizcaino L., Garrido Abellan F., Cubero Pablo M.J. \& Perales A. (1995). - Immunoprophylaxis of caprine contagious agalactia due to Mycoplasma agalactiae with an inactivated vaccine. Vet. Rec., 137 (11), 266-269. https://doi.org/10.1136/vr.137.11.266.

13. Chessa B., Pittau M., Puricelli M., Zobba R., Coradduzza E., Dall’Ara P., Rosati S., Poli G. \& Alberti A. (2009). - Genetic immunization with the immunodominant antigen P48 of Mycoplasma agalactiae stimulates a mixed adaptive immune response in BALBc mice. Res. Vet. Sci., 86 (3), 414-420. https://doi.org/10.1016/ j.rvsc.2008.09.010.

14. European Commission (EC) (2016). - Regolamento (UE) 2016/429 del Parlamento Europeo e del Consiglio del 9 marzo 2016 relativo alle malattie animali trasmissibili e che modifica e abroga taluni atti in materia di sanità animale («normativa in materia di sanità animale»). Off. J. Eur. Union, L 84, 208 pp. Available at: https://eurlex.europa.eu/legal-content/IT/TXT/PDF/?uri=CELEX:32016R0429 (accessed on 10 October 2019).

15. Anon (2014). - Notifiable diseases: Contagious agalactia pathogen confirmed in Wales. Vet. Rec., 175 (19), 468. https://doi.org/10.1136/vr.g6771. 
16. Ministerio de Agricultura y Pesca, Alimentación y Medio Ambiente (MAPA) (2018). - Programa nacional voluntario de vigilancia, control y erradicación de la agalaxia contagiosa ovina y caprina 2018-2020. MAPA, Madrid, Spain, PN AC 2018-2020, 17 pp. Available at: www.mapa.gob.es/fr/ganaderia/temas/sanidad-animalhigiene-ganadera/programaagalaxiacontagiosa20182020ver4def_tcm36-437638.pdf (accessed on 7 March 2019).

17. Poumarat F., Gautier-Bouchardon A.V., Bergonier D., Gay E. \& Tardy F. (2016). - Diversity and variation in antimicrobial susceptibility patterns over time in Mycoplasma agalactiae isolates collected from sheep and goats in France. J. Appl. Microbiol., 120 (5), 1208-1218. https://doi.org/10.1111/jam.13083.

18. Bergonier D., Blaziot J., Larricq J.M., Ticoulet D., Arranz J.M. \& Berthelot X. (2010). - Contagious agalactia in the south-west of France: epidemiological situation, control measures and research work in progress. Bull. GTV, 56, 35-46. Available at: www.cabdirect.org/cabdirect/abstract/20113032887 (accessed on 14 August 2019).

19. Anon (2010). - A

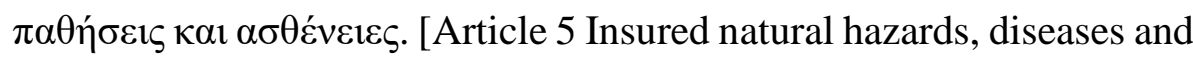

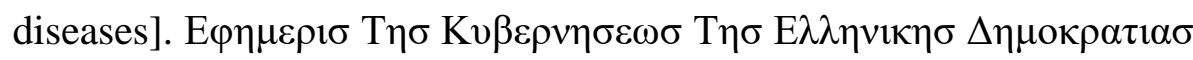
20 [Newspaper of the Government of Hellenic Republic], September 2010, 3377. 